\title{
Cdk5 Suppresses the Neuronal Cell Cycle by Disrupting the E2F1-DP1 Complex
}

\author{
Jie Zhang, Huifang Li, Odessa Yabut, Haley Fitzpatrick, Gabriella D’Arcangelo, and Karl Herrup \\ Department of Cell Biology and Neuroscience, Rutgers, The State University of New Jersey, Piscataway, New Jersey 08854
}

Neurons that reenter a cell cycle after maturation are at increased risk for death, yet the mechanisms by which a normal neuron suppresses the cycle remain mostly unknown. Our laboratory has shown that cyclin-dependent kinase 5 (Cdk5) is a potent cell cycle suppressor, and we report here on the molecular basis of this activity. Cell cycle suppression by Cdk5 requires its binding to the p35 activator protein. The related p 39 and p 25 proteins cannot serve as substitutes. Unexpectedly, Cdk 5 enzymatic activity is not required to perform this function. Rather, the link to cell cycle regulation is made through the formation of a previously unknown complex consisting of the p35-Cdk5 dimer and E2F1. Formation of this complex excludes the E2F1 cofactor, DP1, thus inhibiting E2F1 binding to the promoters of various cell cycle genes. This anti-cell cycle activity is most likely a neuroprotective function of Cdk5.

\section{Introduction}

Neuronal populations at risk for death in a variety of neurodegenerative diseases show evidence of an unscheduled attempt at cell division. The correlation between neuronal cell cycle and cell death is robust; it has been observed in a variety of human diseases including ataxia-telangiectasia, Parkinson's disease, and Alzheimer's disease ( $\mathrm{AD}$ ). In $\mathrm{AD}$, cell cycle events have been described early in the disease process, not merely during end-stage illness (Yang et al., 2003, 2006). Thus, we have argued that suppressing the cell cycle is a constant challenge for a differentiated nerve cell (Herrup and Yang, 2007) and that insights into the mechanism by which neuronal cell cycle regulation is achieved carry significant clinical relevance.

In most dividing cells, the cycle begins when an activating member of the E2F family of transcription factors (E2F1-3) dissociates from the tumor suppressor protein retinoblastoma, RB (Trimarchi and Lees, 2002). Once freed, E2F1 binds to E2F response elements in the promoters of a variety of cell cycle protein genes and activates them, driving the cycle. This activation requires the association of E2F1 with its cofactor, DP1. We have sought to understand the factors that maintain a mammalian neuron in its "postmitotic" state. Recently, we have discovered the participation of Cdk5 in this process (Cicero and Herrup, 2005; Zhang et al., 2008).

Unlike traditional Cdks, ectopic expression of Cdk5 in either mammalian or yeast cells does not promote cell cycle progression (Meyerson et al., 1992; van den Heuvel and Harlow, 1993). Cdk5 activity relies on two unique activator proteins - p35 and p39that are structurally similar to traditional cyclins yet share no

Received Nov. 12, 2009; revised Feb. 11, 2010; accepted Feb. 19, 2010.

This work was supported by National Institutes of Health Grant NS20591. We acknowledge the generosity of the many laboratories that shared reagents with us. Too numerous to list here, they are described in Materials and Methods.

Correspondence should be addressed to Karl Herrup, Department of Cell Biology and Neuroscience, Rutgers, The State University of New Jersey, 604 Allison Road, Piscataway, NJ 08854-8082. E-mail: herrup@biology.rutgers.edu. DOI:10.1523/JNEUROSCI.5628-09.2010

Copyright $\odot 2010$ the authors $\quad 0270-6474 / 10 / 305219-10 \$ 15.00 / 0$ homology at the amino acid level. Cdk5 phosphorylation of numerous substrates plays a pivotal role in many processes of the CNS, including neuronal migration and differentiation during development, as well as synaptic function in the adult (for review, see Cruz and Tsai, 2004; Cheung and Ip, 2007; Hawasli and Bibb, 2007). Cdk5 also plays a crucial role in neuronal cell cycle control. In knock-out animals, the loss of Cdk5 leads to a failure of cell cycle suppression both in vivo and in vitro. Cell cycle activity detected in $C d k 5^{-1-}$ neurons includes the abnormal expression of cell cycle proteins such as cyclin D, cyclin A, and PCNA (proliferating cell nuclear antigen) as well as 5-bromo-2-deoxyuridine (BrdU) incorporation (Cicero and Herrup, 2005). Additional studies suggest that nuclear localization of Cdk5 is an important feature of this activity (Zhang et al., 2008); however, the molecular mechanism behind the cell cycle suppression remains unknown.

We report here that Cdk5 uses an unorthodox noncatalytic method to block the cycle and that this mechanism is effective in primary neurons as well as in neuronal cell lines. Cell cycle suppression is achieved by the assembly of a three-protein complex that includes Cdk5, p35, and the cell cycle activator, E2F1. When sequestered in this complex, E2F1 cannot bind its normal DNA binding partner, DP1. In this way, the formation of the E2F1Cdk5-p35 complex in the nucleus prevents the advance of the cell cycle.

\section{Materials and Methods}

Antibodies and chemical reagents. Antibodies against (phospho-Ser10)p27, p27, $\beta$-actin, p21, Cdk5(C8), Cdk5(J3), GFP(B2), GFP(FL), p35(C19), Lamin B1, cyclin A, E2F1(KH95), and anti-HA were from Santa Cruz Biotechnology. Anti-Myc was from Sigma-Aldrich. Rat-anti-BrdU was bought from Abcam. Secondary antibodies used for immunocytochemistry were as follows: goat anti-mouse Alexa 488 and 594, goat anti-rat Alexa 488 and 594, and goat anti-rabbit Alexa 488 and 594 (Invitrogen). Phospho-histone $\mathrm{H} 3$ antibody was purchased from Millipore.

Cell culture and synchronization. N2a cells were bought from American Type Culture Collection. Mouse embryonic fibroblasts were isolated and cultured as described previously (Kotake et al., 2005). Cells were syn- 
chronized in $G_{0}$ phase by serum deprivation for $48-76 \mathrm{~h}$ in medium supplemented with $0.1 \%$ fetal bovine serum or $0.1 \%$ calf serum. To induce reentry into the cell cycle, cells were transferred to medium containing $10 \%$ fetal bovine serum or $10 \%$ calf serum.

Animals. A colony of $C \mathrm{dk} 5^{-/+}$mice was maintained on a mixed (C57BL/6J $\times 129 / \mathrm{S} 1)$ background (Gilmore et al., 1998). Homozygous $C d k 5^{-\prime-}$ mice are not viable, so mutant embryos were produced by intercrossing heterozygous $C d k 5^{-1+}$ mice. Timed pregnancies were established from these matings; the date of appearance of a vaginal plug was considered embryonic day 0.5 (E0.5). Embryos were taken at E16.5 for either cortical cultures or histology. A colony of $E 2 f 1^{-/+}$ mice was maintained on a mixed (C57BL/6J × 129/ S4) background (Field et al., 1996).

Primary neuronal cultures. Embryonic cortical neurons were isolated by standard procedures. For Cdk5-deficient cultures, all embryos from a $\mathrm{Cdk5}^{+/-} \times \mathrm{Cdk5}^{+/-}$mating were harvested and treated separately. Isolated E15.5 embryonic cerebral cortices from $C d \mathrm{k5}^{-/-}$or $E 2 f 1^{-1-}$ were treated with $0.25 \%$ trypsin-EDTA and dissociated into single cells by gentle trituration. Cells were suspended in Neurobasal medium supplemented with B27 and $2 \mathrm{~mm}$ glutamine, and then plated on coverslips coated with poly-L-lysine $(0.05 \mathrm{mg} / \mathrm{ml})$ and laminin $(5 \mu \mathrm{g} / \mathrm{ml})$. All cultures were grown for a minimum of $5 \mathrm{~d}$ in vitro (DIV) before any treatment.

Constructs and plasmids. The generosity of many laboratories allowed us to assemble a majority of the vectors used in this study. pCMV-p35, pCMV-P39, and pCMV-Cdk5 were from Dr. Li-Huei Tsai (Massachusetts Institute of Technology, Cambridge, MA) (Lee et al., 1996). Myc-CyclinA1 was from Dr. Jonathon Pines (University of Cambridge, Cambridge, UK) (den Elzen and Pines, 2001). HA-cyclinD1 and HA-cyclin D1(T286A) were from Dr. Sarah J. Freemantle (Dartmouth Medical School, Hanover, NH) (Spinella et al., 1999). Myc-CyclinD2 and MycCyclinD2 (T280A) were from Dr. Keiichi I. Nakayama (Kyushu University, Fukuoka, Japan) (Susaki et al., 2007). pBIFC-YN-173; pBIFC-YC-155; pBiFC-YN-Jun and pBiFC-YC-Fos were from Dr. Chang-Deng Hu (Purdue University, West Lafayette, IN) (Hu and Kerppola, 2003; Hu et al., 2002).

We constructed additional vectors as follows. pEGFP-C1 was bought from Clontech. The NLS (from simian virus large T-antigen) and NES [from the dominant nuclear export signal of MAP (mitogen-activated protein) kinase kinase] signal sequences were added into PEGFP-C1 or PECFP-C1 vector after opening the vector with an NheI/AgeI double digest. All inserts were amplified by PCR and inserted into pEGFP-C1NLS. Site-direct mutation of Cdk5 on S159T and D144N (kinase dead) was performed by QuikChange Site-Directed Mutagenesis kit (Stratagene). E2F1 and DP1 were inserted into PECFP-NES.

Bimolecular fluorescence complementation. pBiFC-YN177 and pBIFCYC155 were used in the bimolecular fluorescence complementation systems (Hu et al., 2002). Cdk5 and Cdk5(S159T) were inserted into pBiFC-YN177 to generate pBIFC-YN-177-Cdk5 or pBIFC-YN-177-Cdk5(S159T) vector. Sequences encoding p35 were inserted into pBIFC-YC155 to generate pBIFC-YC155-P35. For analysis of fluorescence complementation, specific YN and YC plasmids were transfected into $C d k 5^{-/-}$primary cultured neurons (DIV 5). Forty-eight hours after transfection, cells were fixed and then examined with a Zeiss LSM510 Meta confocal laser-scanning microscope. pBiFC-YN-Jun and pBiFC-YC-Fos plasmids were used as positive controls (Hu et al., 2002).

In utero electroporation. For in utero electroporation (IUE), expression plasmids under the control of the CAG promoter were used. IUE was conducted as previously described (Saito and Nakatsuji, 2001). Briefly, 3 $\mu l$ of plasmid mix containing Fast Green dye (Sigma-Aldrich) was injected and electroporated into the lateral ventricle of E14.5 embryos using the ElectroSquarePorator ECM 830 (BTX) set at five $50 \mathrm{~ms}$ pulses of $40 \mathrm{~V}$ with $950 \mathrm{~ms}$ intervals. For BrdU (Sigma-Aldrich) labeling, electroporated pregnant dams were treated with $100 \mu \mathrm{g} / \mathrm{g}$ BrdU by intraperitoneal injection $20 \mathrm{~h}$ after IUE. Four hours later, electroporated embryonic brains were dissected and fixed overnight in $4 \%$ PFA at $4^{\circ} \mathrm{C}$, and then placed in 30\% sucrose $/ 1 \times$ PBS mix for cryoprotection. Brains were frozen in OCT (Tissue-Tek) and sectioned coronally at 12-20 mm.

Electrophoretic mobility shift assays. N2a cells were cultured in DMEM and transfected with various Cdk5 constructs. Nuclear extracts were collected for electrophoretic mobility shift assay (EMSA). Double-stranded oligonucleotides used as a labeled DNA probe included the E2F-binding site ATTTAAGTTTCGCGCCCTTTCTCAA. The mutant E2F bindingdeficient oligonucleotide was ATTTAAGTTTCGATCCCTTTCTCAA (Cao et al., 1992). For competition experiments, a 100-fold molar excess of unlabeled oligonucleotides was added before the addition of labeled probe. The electrophoretic mobility shift assays were performed with the LightShift Chemiluminescent EMSA kit (Pierce) according the manufacturer's instructions.

Immunocytochemistry and BrdU incorporation. At the appropriate time, cultures were rinsed once with PBS and then exposed to $4 \%$ paraformaldehyde in $0.1 \mathrm{M}$ phosphate buffer for $30 \mathrm{~min}$ at room temperature followed by three rinses with PBS. Immunohistochemistry of cell cultures was done without antigen retrieval. For BrdU labeling, the cells were cultured normally or serum starved for $48 \mathrm{~h}$ followed by $12 \mathrm{~h}$ of serum add back. Four hours before the end of the experiment, $10 \mu \mathrm{M} B r d U$ was added to the media. The cells were then fixed, and DNA was hydrolyzed by exposing the cells to $2 \mathrm{~N} \mathrm{HCl}$ for $10 \mathrm{~min}$. Specimens were then neutralizing in $0.1 \mathrm{~m}$ sodium borate, $\mathrm{pH}$ 8.6, for $10 \mathrm{~min}$ and rinsed extensively in PBS $(3 \times)$ for $45 \mathrm{~min}$ before treatment with blocking reagent. Nonspecific antibody binding was blocked by exposing the fixed cells to $5 \%$ normal goat serum in $0.1 \%$ Triton $\mathrm{X}-100$ for $1 \mathrm{~h}$ before application of the primary antibody.

Western blotting, coimmunoprecipitation. Dissected tissues or harvested cells were homogenized in 1:5 (w/v) ice-cold lysis buffer [1\% Triton X-100, $20 \mathrm{~mm}$ Tris- $\mathrm{HCl}, \mathrm{pH}$ 7.5, $150 \mathrm{~mm} \mathrm{NaCl}$, with protease inhibitor mix (Roche)]. The samples were centrifuged at $12,000 \times g$ for 


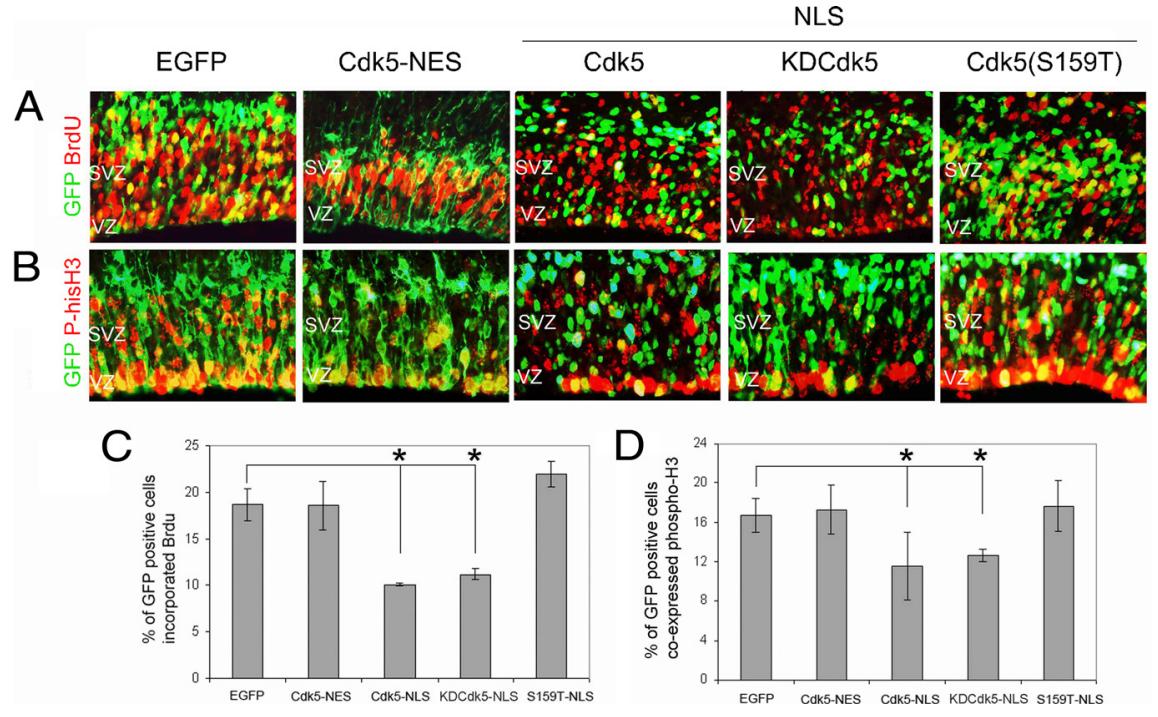

Figure 2. Nuclear Cdk5 is an effective cell cycle suppressor in vivo as well as in vitro. GFP, GFP-Cdk5-NLS, kinase-dead GFPKDCdk5-NLS, and p35 binding-deficient GFP-CDk5(S159T)-NLS were introduced into the ventricular zone of the E14.5 embryo by IUE and subjected to short-term BrdU labeling at E15.5. $\boldsymbol{A}, \boldsymbol{B}$, Representative images of $10 \mu \mathrm{m}$ sections obtained from IUE-treated embryos immunostained for either BrdU $(\boldsymbol{A})$ or phospho-histone H3 (P-hisH3) (B). C, D, Quantification of BrdU/GFP $(\boldsymbol{C})$ and phospho-histone H3/GFP $(\boldsymbol{D})$ double-labeled neurons. ${ }^{*} p<0.05$ by ANOVA. Values are mean $\pm \mathrm{SEM}$.
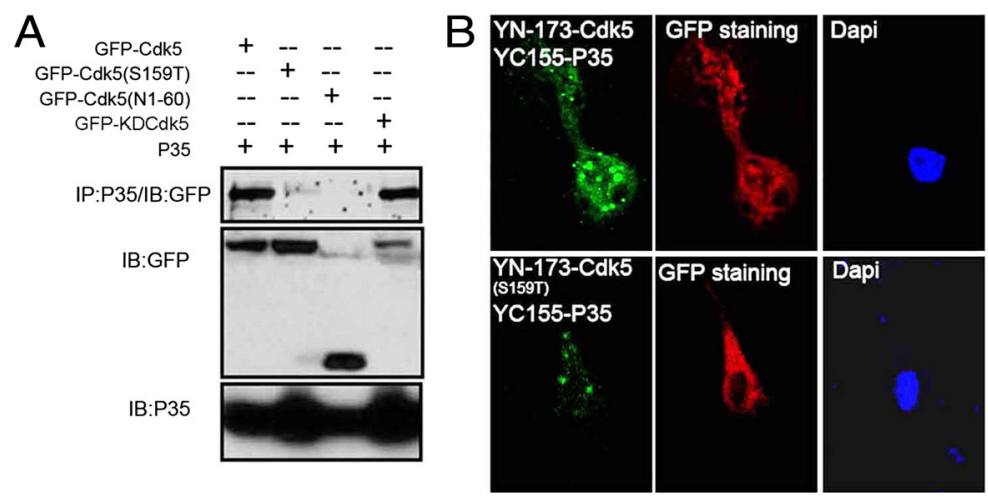

Figure 3. $\mathrm{p} 35$ is bound to $C \mathrm{dk} 5$ in the nucleus as well as the cytoplasm. $\boldsymbol{A}$, Coimmunoprecipitation experiments show the interaction of p35 with Cdk5-GFP or KDCdk5-GFP, but not to Cdk5(S159T)-GFP or Cdk5(N1-60)-GFP in N2a cells. B, BiFC fluorescence signal generated in the neuronal nucleus from the interaction of YN173-Cdk5 with YC155-p35 (top row). When the p35 binding-deficient (S159T) mutation is used (bottom row), this signal is lost. In both situations, immunostaining with anti-GFP antibody reveals the wide cellular distribution of the exogenous proteins. DAPI was used as a nuclear counterstain.

$20 \mathrm{~min}$ at $4^{\circ} \mathrm{C}$. The supernatant was collected and total protein levels were measured by a micro BCA protein assay kit (Pierce). Fractionation of cells into cytoplasmic and nuclear components was accomplished with an NER-mammalian kit according to the manufacturer's instructions (Pierce/Thermo Fisher Scientific).

For Western blots, lysates were separated with SDS-PAGE and electrophoretically transferred onto nitrocellulose membranes. Membranes were blocked with 5\% nonfat milk in TBST and probed with primary antibodies in blocking buffer, followed by treatment with HRP-linked secondary antibodies and ECL Western blotting detection reagents (Pierce/Thermo Fisher Scientific). The intensity of immunoreactive bands was quantified using NIH Image.

For immunoprecipitation (IP), cell lysates were incubated with antibody shown in the IP blot at $4^{\circ} \mathrm{C}$ for $90 \mathrm{~min}$, followed by additional incubation with protein G-Sepharose (GE Healthcare) for $90 \mathrm{~min}$. The beads were washed five times with ice-cold PBS, and bound proteins were analyzed by SDS-PAGE and immunoblot analysis.

Statistical analysis. All data were obtained from at least three different preparations. Values are mean \pm SEM. Data analysis was performed by one-way ANOVA, and $p<0.05$ was considered significant.

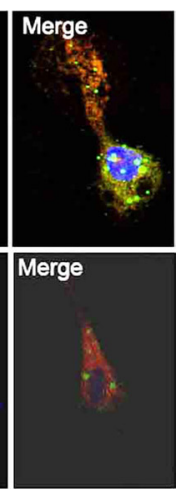

\section{Results}

Cdk5 blocks cycle reentry: localization dependence and activity independence Our previous studies showed that the presence of Cdk5 in the nucleus is important for it to stop the cell cycle in cultured neurons and cell lines (Zhang et al., 2008). To explore this novel role of Cdk5 more thoroughly, we conducted a series of experiments in the $\mathrm{N} 2 \mathrm{a}$ neuroblastoma cell line. N2a cells in log-phase growth were transfected with a variety of expression constructs, growth arrested in low serum for $48 \mathrm{~h}$, and then restored to normal growth medium to mimic the conditions of cell cycle reactivation in postmitotic neurons. To study the effect of Cdk5 in different cellular compartments, we generated green fluorescent protein (GFP)tagged expression constructs directing the kinase specifically in the nucleus (GFPCdk5-NLS), cytoplasm (GFP-Cdk5-NES), or in both compartments (GFP-Cdk5). As controls, we used three GFP-only constructs as follows: (1) native GFP, (2) GFP-NLS, and (3) GFP-NES. BrdU incorporation was used to examine the effect of these constructs on cell proliferation. When control plasmids were used, the percentage of double-labeled GFP-BrdU cells was 50\% (Fig. 1A,B). A similar result was obtained using GFP-Cdk5-NES. However, the expression of nondirected Cdk5 (GFP-Cdk5) and nuclear Cdk5 (GFP-Cdk5-NLS) reduced the percentage of double-labeled cells to $\sim 10 \%$, suggesting that the cell cyclearresting activity of $\mathrm{Cdk} 5$ is performed in the nucleus.

To investigate whether the kinase activity of Cdk5 is required for this activity, we conducted similar experiments using mutagenized Cdk5 constructs that were deficient in kinase activity. In one of these constructs, the serine at position 159 was substituted by a threonine [Cdk5(S159T)]. This mutation blocks the ability of Cdk5 to bind its activator p35 resulting in a loss of kinase activity (Tarricone et al., 2001). We found that Cdk5(S159T) was unable to block BrdU incorporation regardless of its localization (Fig. 1A,B). We also examined a Cdk5 mutant in which the ATP binding site, well known to be essential for kinase activity, was mutated resulting in a "kinase-dead" Cdk5 (KDCdk5) (Tsai et al., 1994; Nikolic et al., 1996). Surprisingly, this construct, no matter where it was targeted (GFP-KDCdk5, GFP-KDCdk5-NLS or GFP-KDCdk5-NES), behaved similarly to its wild-type $\mathrm{Cdk} 5$ counterparts in our BrdU incorporation assay (Fig. $1 A, B$ ).

To confirm the results of our BrdU measurements, we used phospho-histone $\mathrm{H} 3$, a marker of cells in the $\mathrm{M}$ phase of the cell cycle. As shown in Figure 1, $C$ and D, expression of Cdk5-NLS and KDCdk5-NLS decreased phospho-histone H3 labeling by approximately one-half compared with control. Cdk5-NES and Cdk5(S159T)-NLS overexpression had no effect on the phospho- 
histone $\mathrm{H} 3$ staining. These results are consistent with the BrdU data described above. Together, we interpret these results to mean that Cdk5 does not need its catalytic activity to function as a cell cycle suppressor, but it does need to bind to its activator, p35.

To determine whether these observations applied equally in vivo, we used the technique of IUE to introduce GFP or GFP-tagged Cdk5 into neural progenitor cells lining the ventricular zone of the E14.5 mouse embryo. Dividing cells were marked by BrdU incorporation and phospho-histone $\mathrm{H} 3$ labeling $1 \mathrm{~d}$ after IUE. As shown in Figure 2, $A$ and $C$, when GFP alone was expressed in progenitor cells, the percentage of GFP/BrdU doublepositive cells represented $\sim 17 \%$ of the total transfected population. This number changed little when GFP was trafficked to the nucleus or to the cytoplasm, or when cytoplasmic Cdk5 (GFP-Cdk5-NES) was expressed. In contrast, nuclear Cdk5 (GFP-Cdk5-NLS) significantly decreased the double-labeled percentage to $\sim 8 \%$. Similar results were obtained when the percentage of phospho-histone H3/GFP cells was calculated as a marker of cell division (Fig. $2 B, D$ ). These data demonstrate that $\mathrm{Cdk} 5$ can act as a neuronal cell cycle suppressor in vivo.

The role of the kinase activity of Cdk5 was also verified in vivo. We conducted IUE experiments with both GFP-KDCdk5NLS and GFP-Cdk5(S159T)-NLS. Similar to the results in N2a cells, when KDCdk5NLS was electroporated into the E14.5 mouse embryo the percentage of BrdU/ GFP or phospho-histone H3/GFP double-labeled cells decreased significantly compared with control, whereas Cdk5(S159T)-NLS showed no effect (Fig. 2). Thus, our in vivo and in vitro data are in agreement and suggest that, to suppress the cell cycle, Cdk5 must be present in the nucleus, it must be able to bind to p35, but it does not need to be catalytically active.

\section{The role of p35 in cell cycle suppression}

We conducted biochemical coimmunoprecipitation experiments to elucidate the role of p35 in cell cycle suppression. GFPtagged Cdk5 constructs were coexpressed with p35 in N2a cells. The interaction of Cdk5 with p35 was strong in this assay (Fig. $3 A$ ), but it was prevented either by the truncation of the $\mathrm{C}$ terminus of Cdk5 (N1-60 construct, lane 3) or by the S159T mutation of the p35 binding site (lane 2). The KD mutation of Cdk 5 bound p35 as avidly as the wild-type protein (lane 4), a result that is consistent with its undiminished ability to suppress the cell cycle. To determine whether Cdk5 forms a complex with p35 in the nucleus, where the cell cycle-arresting activity was observed, we used bimolecular fluorescence complementation (BiFC) (Kerppola, 2006). Reconstituted GFP fluorescence produced by Cdk5-p35 binding appeared to be strong in the cytoplasm, but it was also detectable in the nucleus where it was colocalized with the $4^{\prime}, 6^{\prime}$ diamidino-2-phenylindole (DAPI) counterstain. As expected, immunostaining with a GFP antibody that detects both halves of the GFP molecule revealed a uniform pattern of labeling, emphasizing the specific nature of the BiFC fluorescence (Fig. 3B). A p35 binding-deficient $\mathrm{Cdk} 5$ (Cdk5S159T) was used as a negative control for BiFC (Fig. 3B, bottom row). These data demonstrate that the Cdk5-p35 complex exists in the nucleus as well as in the cytoplasm.

The Cdk5 kinase can be activated by either of two cyclin-like proteins: p35 or p39. The p35 protein can also be cleaved to generate a p25 isoform plus a smaller p10 fragment; p25 is known to stabilize Cdk5 and to lead to its hyperactivation. The data thus far show that cell cycle suppression by Cdk5 requires p35. To investigate the role of these other potential binding partners, we expressed GFP-tagged p35, p39, p10, and p25 with nuclear or cytoplasmic trafficking signals in N2a cells (Fig. 4A,C). Consistent with our hypothesis, p35 expression in the nucleus decreased the percentage of double-BrdU-labeled cells within the GFP-positive population by more than fivefold. In contrast, neither p25, p39, nor p10 had any effect on BrdU incorporation even when these proteins were trafficked to the nucleus. All four binding proteins, when trafficked to the cytoplasm, had no effect compared with a GFP-NES control (Fig. 4B,D). From this result, we conclude that neither p25, p10, nor p39 is able to replace p35 in the cell cyclesuppressing activity of Cdk5. 
A
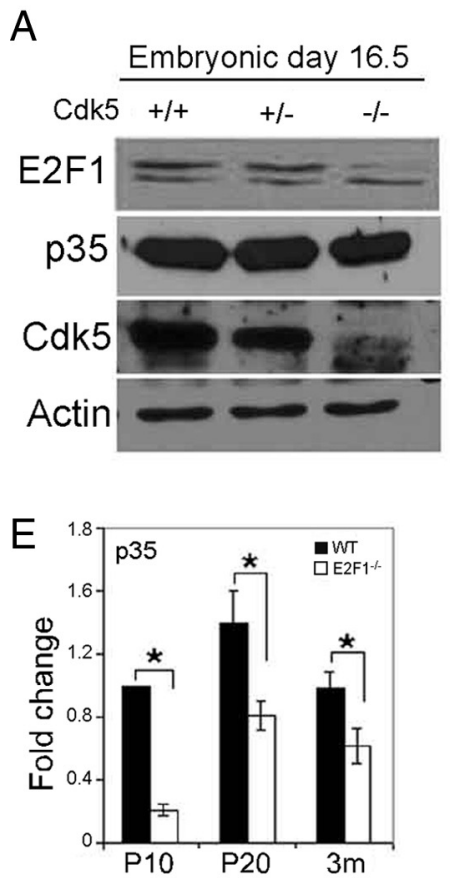

B

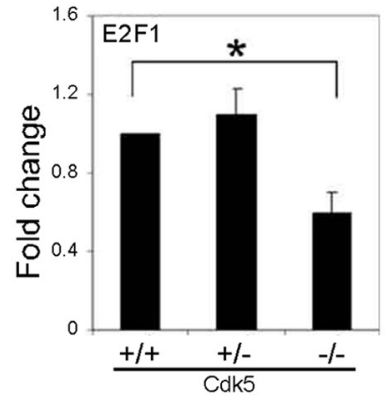

C

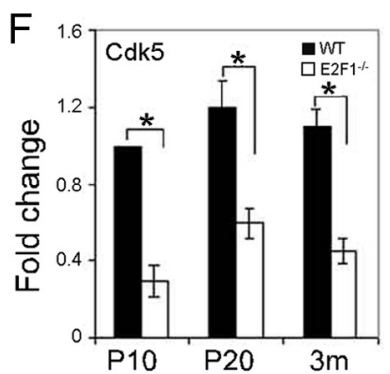

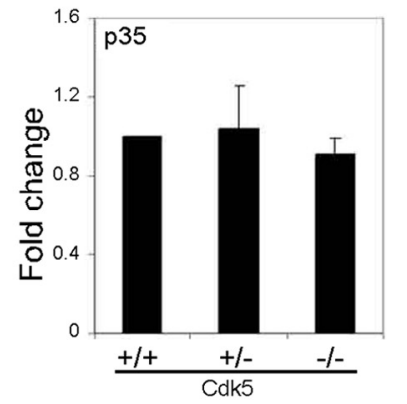

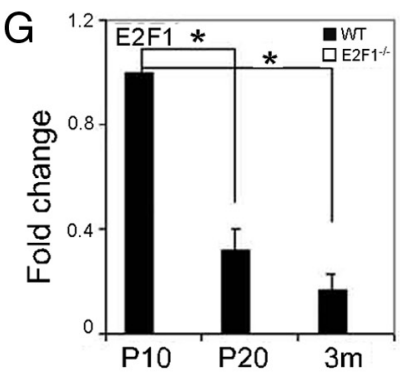

$\mathrm{D}$

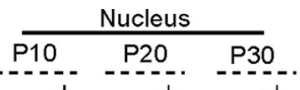

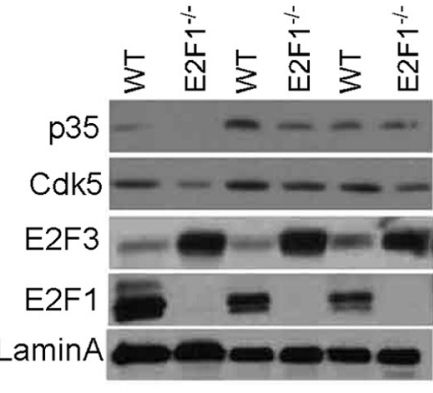

$\mathrm{H}$

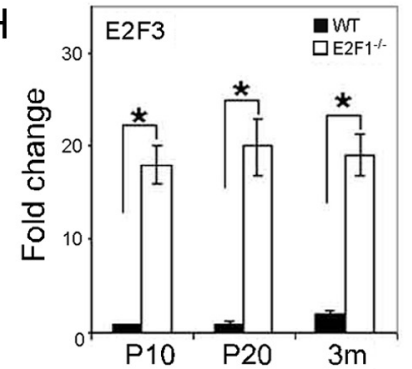

Figure 5. The relationship between E2F1 and Cdk5 expression in vivo. $A, E 2 F 1$ and p35 levels in the E16.5 cortex of three genotypes: $C d k 5^{-1-}, C d k 5^{-1+}$, and $C d k 5^{+/+}$mice. B, C, Quantification of the data shown in $\boldsymbol{A}$ for E2F1 (B) and p35 (C).D, Nuclear Cdk5, p35, and E2F3 levels in E2f1 ${ }^{-1-}$ and wild-type cortex from the mice at P10, P20, and 3 months of age. $\boldsymbol{E}-\boldsymbol{H}$ represent the quantitative analysis of the data shown in $\boldsymbol{D}$. ${ }^{*} p 0.05$ by ANOVA. Values are mean \pm SEM.

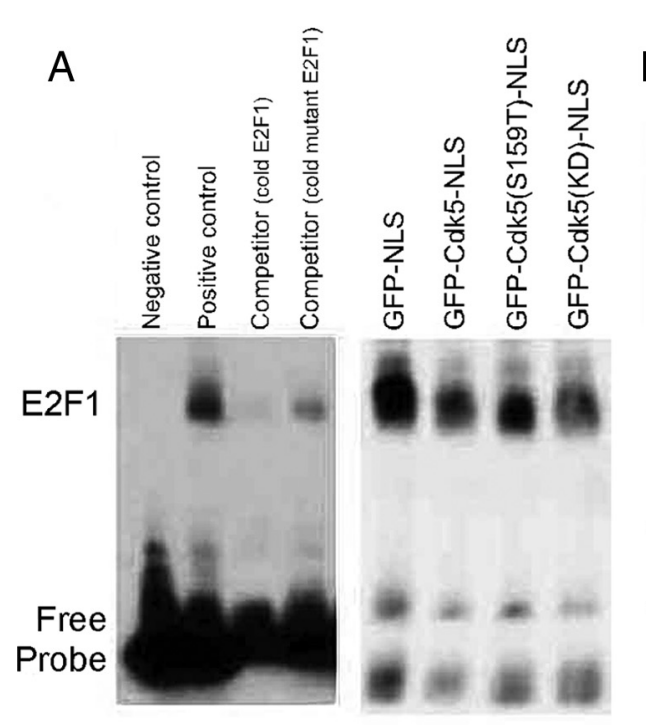

B

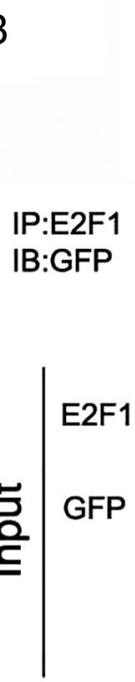

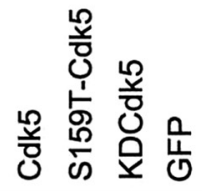
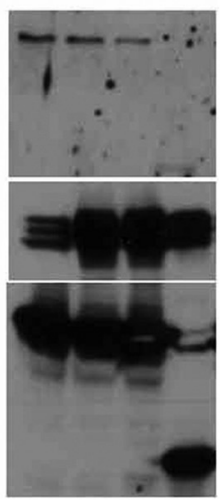

C

\begin{tabular}{|c|c|c|c|c|}
\hline 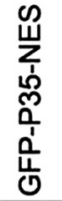 & 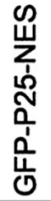 & 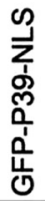 & 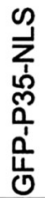 & 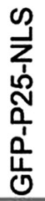 \\
\hline
\end{tabular}

IP:E2F1

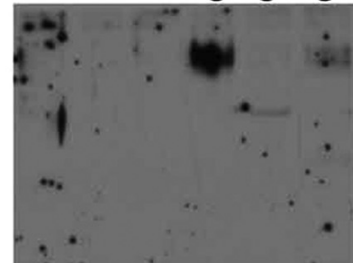

CFP-E2F1

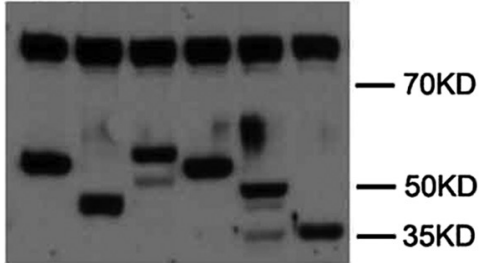

Figure 6. Nuclear Cdk5 interacts physically with E2F1 and blocks its access to its normal DNA binding sites. $A$, EMSA reveals changes in the DNA binding ability of E2F1 after overexpression of different Cdk5 constructs. The shifted probe caused by E2F1 binding is indicated by the band labeled E2F1. B, N2a cells were cotransfected with E2F1 plus GFP-Cdk5, GFP-Cdk5(S159T), GFP-KDCdk5, or native GFP. After immunoprecipitation with E2F1, blots were immunostained with GFP antibody. Note that wild-type Cdk5, Cdk5(S159T), and KDCdk5 were all able to pull down E2F1 (top blot). The bottom blot is the input lysates Western blotted before immunoprecipitation. C, N2a cells were cotransfected with GFP-P35-NES, GFP-P25-NES, GFP-P39-NLS, GFP-P35-NLS, GFP-P25-NLS, GFP-P10-NLS with CFP-E2F1. After immunoprecipitation with E2F1, blots were immunostained with GFP antibody. Note that E2F1was able to pull down only nuclear p35.

Cdk5 inhibits promoter occupancy at E2F1-responsive genes

To further investigate the mechanism by which the Cdk5-p35 complex suppresses the cell cycle, we turned our attention to the transcription factor E2F1, a major factor regulating the $G_{0} / G_{1}$ - to $\mathrm{S}$-phase transition at the beginning of each new cell cycle (Trimarchi and Lees, 2002). We explored the possibility that E2F1 might physically or functionally interact with Cdk5. We first examined the levels of E2F1 in the E16.5 Cdk5 $5^{-l-}$ cortex (Fig. 5A) and found that they were significantly lower than in wild type (Fig. $5 B)$; the levels of p35 did not change dramatically (Fig. 5A,C). Conversely, the levels of both p35 and Cdk5 were significantly lower in $E 2 \mathrm{fl}^{-1-}$ cortex at all postnatal ages examined [postnatal day 10 (P10), P20, and P90] (Fig. 5D-F). Note that the levels of E2F1 decreased over this age span in wild-type animals (Fig. 5G), 
A $123 \quad 4 \quad 566788910111213$

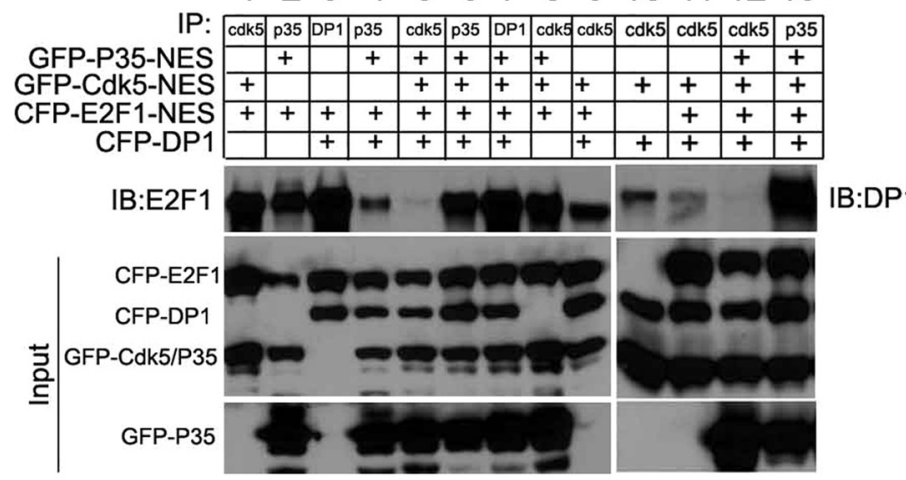

B

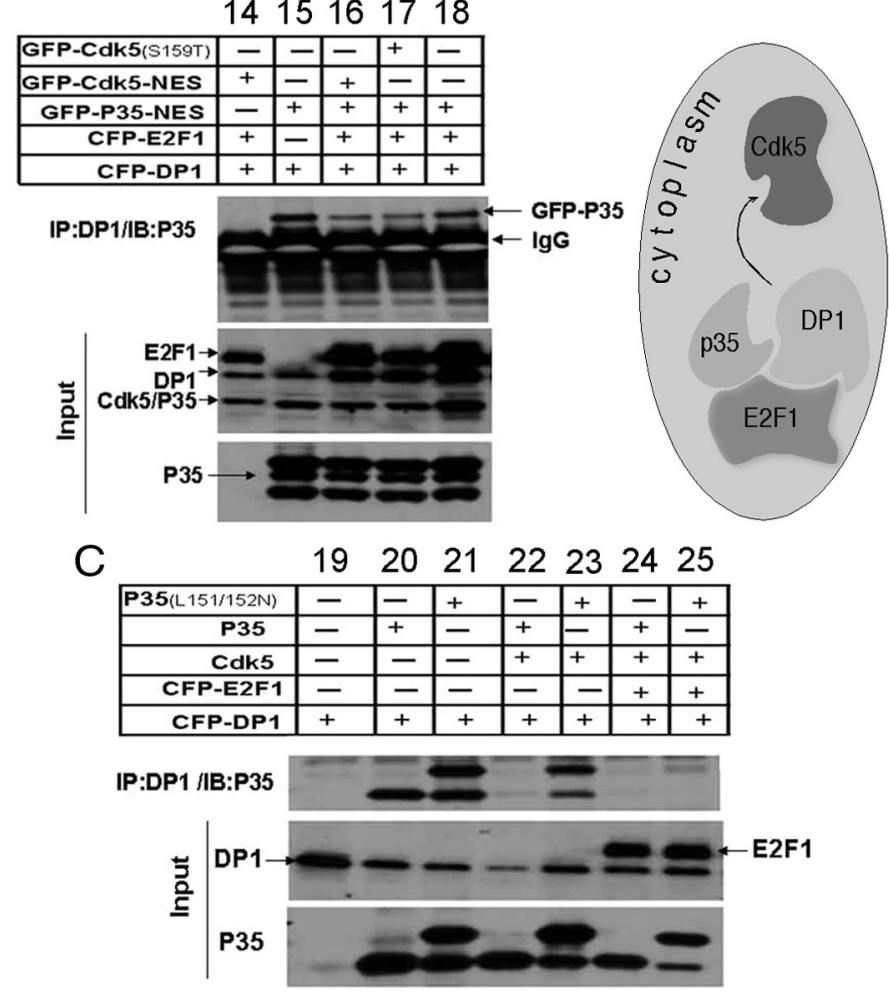

D

\begin{tabular}{|c|c|c|c|c|}
\hline $\begin{array}{l}\text { Protein } \\
\text { expression }\end{array}$ & IP: & IB: & Binding & Lane \\
\hline E2F1 DP1 & DP1 & E2F1 & + & 3 \\
\hline $\begin{array}{ll}\text { E2F1 } & \text { p35 } \\
\end{array}$ & p35 & E2F1 & + & 2 \\
\hline E2F1 Cdk5 & Cdk5 & E2F1 & + & 1 \\
\hline DP1 135 & DP1 & p35 & + & $15 / 20$ \\
\hline DP1 p35(L151N) & DP1 & p35 & + & 21 \\
\hline DP1 Cdk5 & Cdk5 & DP1 & + & 10 \\
\hline Cdk5 p35 & Cdk5 & p35 & + & not shown \\
\hline \multirow{3}{*}{$\begin{array}{l}\text { E2F1 } \\
\text { DP1 } \\
\text { p35 }\end{array}$} & DP1 & p35 & + & 18 \\
\hline & DP1 & E2F1 & + & not shown \\
\hline & p35 & E2F1 & + & 4 \\
\hline \multirow{3}{*}{$\begin{array}{l}\text { E2F1 } \\
\text { DP1 } \\
\text { Cdk5 }\end{array}$} & Cdk5 & E2F1 & + & 9 \\
\hline & Cdk5 & DP1 & + & 11 \\
\hline & DP1 & E2F1 & + & 14 \\
\hline \multirow{3}{*}{$\begin{array}{l}\text { E2F1 } \\
\text { p35 } \\
\text { Cdk5 }\end{array}$} & Cdk5 & E2F1 & + & 8 \\
\hline & Cdk5 & p35 & + & not shown \\
\hline & p35 & E2F1 & + & not shown \\
\hline \multirow{3}{*}{$\begin{array}{l}\text { DP1 } \\
\text { p35 } \\
\text { Cdk5 }\end{array}$} & DP1 & p35 & - & 22 \\
\hline & DP1 & Cdk5 & - & not shown \\
\hline & Cdk5 & p35 & + & not shown \\
\hline \multirow{3}{*}{$\begin{array}{l}\text { DP1 } \\
\text { p35(L151N) } \\
\text { Cdk5 }\end{array}$} & DP1 & p35 & + & 23 \\
\hline & DP1 & Cdk5 & + & not shown \\
\hline & Cdk5 & p35 & - & not shown \\
\hline \multirow{6}{*}{$\begin{array}{l}\text { E2F1 } \\
\text { DP1 } \\
\text { Cdk5 } \\
\text { p35 }\end{array}$} & Cdk5 & E2F1 & - & 5 \\
\hline & Cdk5 & DP1 & - & 12 \\
\hline & DP1 & E2F1 & + & 7 \\
\hline & p35 & E2F1 & + & 13 \\
\hline & p35 & DP1 & + & $16 / 24$ \\
\hline & Cdk5 & p35 & - & not shown \\
\hline \multirow{2}{*}{$\begin{array}{r}\text { w/p35(L151N) } \\
\text { w/Cdk5(S159T) }\end{array}$} & DP1 & p35 & - & 25 \\
\hline & DP1 & p35 & + & 17 \\
\hline
\end{tabular}

Figure 7. The interactions among DP1, E2F1, Cdk5, and p35 in the cytoplasm. A, N2a cells were cotransfected with two, three, or four of the GFP or CFP chimeric plasmids indicated in the table at the top of the gel. The antibody used for IP is indicated in the top row of the table. The antibodies used to probe the resulting blots were E2F1 (lanes 1-9) and DP1 (lanes 10-13). Probing of the input lysates (bottom gels) reveals the comparable expression of the various constructs. B, Cotransfections of N2a cells were performed as indicated. For all lanes, lysates were immunoprecipitated with DP1 antibody, and the resulting Western blots were probed with antibody against p35. C, Both the antibody used to perform the immunoprecipitation and the one used in the Western blot are indicated at the side of the blot. D, A table summarizing all of the interaction patterns for the four proteins in the cytoplasm. The proteins listed in the first column (protein expression) were cotransfected into N2a cells. The resulting cell lysates were immunoprecipitated (IP) with the antibody listed in the second column. The precipitates were analyzed by Western blots, blotted (IB) with the antibody listed in the third column. The fourth column (Binding) summarizes the IP results: a successful IP is indicated by a " +"; a failure to IP is indicated by a " -." The lane numbers of the gel that document the binding results are indicated in the final column (note: some interactions were duplicated on the gels illustrated; some interactions are notated here but were observed on gels that are not shown). The diagram in the center of the figure illustrates our model for the behavior of the four proteins when coexpressed in the cell cytoplasm.

whereas the levels of $\mathrm{E} 2 \mathrm{~F} 3$ increased dramatically in $E 2 \mathrm{fi}^{-1-}$ cortex (Fig. 5H).

To determine whether Cdk5 and p35 affected the transcription activity of E2F1, we conducted EMSAs using a well characterized E2F1 response element. Wild-type Cdk5, KDCdk5, and Cdk5(S159T) were expressed in the nucleus, and the effects on promoter occupancy were assayed. Cdk5 and KDCdk5 both significantly decreased the E2F1 DNA binding activity, whereas the inactive Cdk5(S159T) had no effect on the EMSA pattern (Fig. $6 A$ ). The E2F1 protein level was not significantly different among these assays (data not shown). These data reveal that $\mathrm{Cdk} 5 \mathrm{can}$ decrease the promoter occupancy of E2F1 and led us to ask whether there was a direct physical interaction between $\mathrm{Cdk} 5$ and E2F1. As shown in Figure 6B, immunoprecipitation of E2F1 pulled down wtCdk5 and KDCdk5 as well as Cdk5(S159T) in N2a cells. To explore this interaction further, we expressed E2F1 to- 


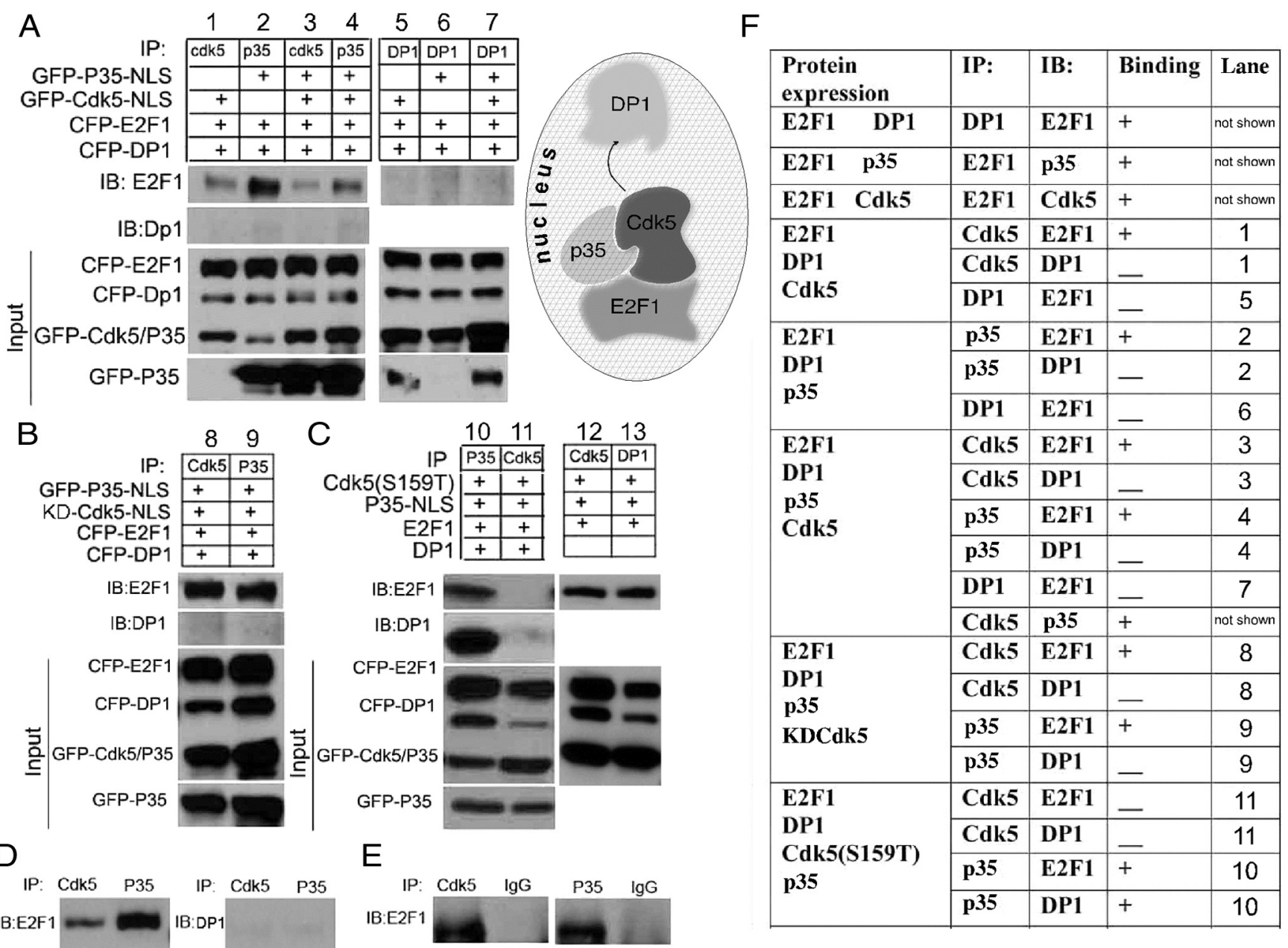

Figure 8. The interactions among DP1, E2F1, Cdk5, and p35 were investigated in the nucleus. A-C, N2a cells were cotransfected with two, three, or four of the GFP or CFP chimeric plasmids indicated in the table at the top of the gel. The antibody used for IP is indicated in the top row of the table. The antibodies used to probe the resulting blots were E2F1 and DP1. Probing of the input lysates (bottom gels) reveals the comparable expression of the various constructs. $\boldsymbol{D}$, Immunoprecipitations of cell lysates of cultured cortical neurons from E16.5 wild-type mice. Neurons were transfected after harvest with Cdk5, DP1, p35, and E2F1 plasmids, and then harvested after $6 \mathrm{~d}$ in vitro. $\boldsymbol{E}$, Immunoprecipitations of brain tissue lysates from adult wild-type mice. $\boldsymbol{F}$, A table summarizing all of the interaction patterns for the four proteins in the nucleus. Note the different partnerships in this environment compared with the cytoplasm (Fig. 8). The proteins listed in the first column (protein expression) were cotransfected into N2a cells. The resulting cell lysates were immunoprecipitated with the antibody listed in the second column. The precipitates were analyzed by Western blots, with the antibody listed in the third column (IB). The fourth column (Binding) summarizes the IP results: a successful IP is indicated by a " + "; a failure to IP is indicated by a " -.." The lane numbers of the gel that document the binding results are indicated in the final column (note: some interactions were observed on gels that are not shown). The diagram in the center of the figure illustrates our model for the behavior of the four proteins when coexpressed in the nucleus.

gether with p35, p39, p25, and p10. As shown in Figure 6C, nuclear p35 bound E2F1, whereas p39, p25, and p10 did not. Interestingly, $\mathrm{p} 35$ was unable to bind $\mathrm{E} 2 \mathrm{~F} 1$ if it was directed to the cytoplasm with an NES. Together with the EMSA promoter occupancy data, a model emerges in which it is the ability to sequester E2F1 that serves as the basis of the cell cycle suppression activity of the $\mathrm{p} 35-\mathrm{Cdk} 5$ complex.

\section{Cdk5-p35 dissociates the E2F1-DP1 complex}

Each E2F family member associates with a member of the DP family to achieve full activity. E2F1 localizes almost exclusively to the nucleus, whereas DP1 alone is found in the cytoplasm; if they are coexpressed, DP1 relocates to the nucleus (supplemental Fig. 1 , available at www.jneurosci.org as supplemental material) (Ishida et al., 2005). Therefore, we investigated the physical interactions among Cdk5, E2F1, DP1, and p35.

First, we examined the interaction among these proteins in the cytoplasm using NES-directed Cdk5 and p35 constructs (Fig. $7 A-C)$. When protein pairs were coexpressed in N2A cells, E2F1 coprecipitated with Cdk5, p35, or DP1 (Fig. 7D, lanes 1-3). However, when all four proteins were coexpressed together, E2F1 was found to bind DP1 and p35, but not Cdk5 (Fig. 7D, lanes 5, 7, 12, $13,16,24)$. Since p35, but not Cdk5, was also found to bind DP1, these data suggest that a tertiary complex exists in the cytoplasm that is composed of E2F1, DP1, and p35; Cdk5 is excluded.

Second, we studied how nuclear localization of Cdk5 and p35 affected the complex. As in the nucleus, we found that E2F1 bound strongly to p35 and also to Cdk5 (Fig. 8F, lanes 1-4). However, DP1 was no longer able to bind E2F1 (lanes 5-7). This suggests that, in the environment of the cell nucleus, Cdk5 partners with p35 to disrupt the interaction of E2F1 and DP1 that is necessary for cell cycle progression. To further explore the significance of this interaction, we conducted experiments using Cdk5(S159T) and KDCdk5. We found that the Cdk5(S159T) mutant, which does not bind p35, was unable to interact with E2F1(lanes 10 and 11), whereas KDCdk5 bound this factor similarly to wild-type Cdk5 (lanes 8 and 9). These data indicate that p35 is required for the formation of a nuclear complex consisting 
of E2F1, p35, and Cdk5, and that this complex formation does not require Cdk5 kinase activity. To further confirm that kinase activity is not needed for Cdk5 cell cycle suppression, N2a cells were transfected with nuclear targeted Cdk5 (GFPCdk5-NLS), and then labeled with BrdU in the presence or absence of the Cdk5 kinase inhibitor, roscovitine. As predicted from the results with the KDCdk5 mutant, pharmacological inhibition of Cdk5 activity had no effect on its cell cycle suppression properties.

The Cdk5-p35-E2F1 complex we have described also exists in vivo. We performed a series of immunoprecipitations from wild-type brain lysates and found that all of the interactions predicted from our in vitro work were detected in this material. First, the Cdk5-p35-E2F1 complex was found in overexpressed primary neurons as shown in Figure 8 D. Second, Cdk5 and p35 can both pull down E2F1 from brain lysates (Fig. $8 E$ ). Finally, a key prediction of our model is that Cdk5 should be unable to suppress the cell cycle in the absence of E2F1. We have previously shown that $E 2 \mathrm{fl}^{-1-}$ neurons spontaneously enter a cell cycle in vitro (Wang et al., 2007). As predicted, a GFP-Cdk5-NLS construct transfected into these E2F1deficient neurons was unable to arrest this process (supplemental Fig. 2, available at www.jneurosci.org as supplemental material). Similarly, if p35 or E2F1 were knocked down with siRNA, Cdk5 was no longer capable of suppressing the cell cycle, even if trafficked to the nucleus (supplemental Fig. 3, available at www.jneurosci.org as supplemental material).

\section{Discussion}

Cell cycle suppression by Cdk5 is achieved through its participation in a multiprotein complex that includes E2F1. The involvement of E2F1 in the mechanics of Cdk5 cell cycle suppression is significant. The E2Fs are a family of transcription factor proteins best known for their role in regulating cell proliferation, differentiation, and apoptosis through transcriptional regulation (Ishida et al., 2001; Müller et al., 2001; Ren et al., 2002; Trimarchi and Lees, 2002). Our EMSA data provide evidence that Cdk5 has the capacity to reduce the transcription of genes with E2F1 response elements. The basis for this inhibition can be found in our studies of the impact of Cdk5 on the association of E2F1 with its cofactor, DP1. It has been shown that, in the absence of DP1, E2F1-dependent transcriptional activation decreases dramatically (Bandara et al., 1993). By binding E2F1 and displacing DP1, wild-type Cdk5 and KDCdk5 both decrease the occupancy of the E2F1 promoter element and hence can function as effective cell cycle suppressor proteins. This is a $\mathrm{p} 35$-dependent process as can be seen from the fact that the Cdk5(S159T) mutant does not affect promoter occupancy and, as would be predicted, does not function as a cell cycle suppressor.

The requirement for $\mathrm{p} 35$ binding in the formation of the complex is intriguing. Although its binding to Cdk5 is well known, its association with E2F1 (Fig. 8) is unexpected. We note that p35 is most similar in size and structure to cyclin A and there is a known cyclin A binding site on E2F1. Furthermore, the binding of cyclin A blocks the ability of E2F1 to support the transcription needed for full cell cycle progression (Mudryj et al., 1991; Krek et al., 1994). Thus, we would predict that the interaction of p35 with E2F1 is through this cyclin A site. Cyclin A and D also can bind with Cdk5 (Xiong et al., 1992; Zhang et al., 1993; Lee et al., 1996; Guidato et al., 1998), but surprisingly the S159T mutation has no effect on this interaction (supplemental Fig. $4 A$, available at www.jneurosci.org as supplemental material). Similarly, the T286A mutation of cyclin D1, which is important for its subcellular localization, binds to Cdk5 as well as its wild-type counterpart. Finally, Cdk5 binding to cyclins D2 and A is very weak (supplemental Fig. 4B, C, available at www.jneurosci.org as supplemental material). Together, these data argue that Cdk5 is not "buffering" other cyclins to block their access to Cdk4 and Cdk6. Rather, it is p35 that is the factor most directly involved in Cdk5 cell cycle suppression.

As the main activator of $\mathrm{Cdk} 5, \mathrm{p} 35$ contains a myristoylation signal motif, which allows it to be anchored to the plasma membrane. Since it is the p10 fragment that contains the myristoylation anchor, cleavage of membrane-bound p35 liberates p25 to the cytoplasm (Nikolic et al., 1998; Patrick et al., 1999). Although this would predict that most p35 would be membrane bound, in cultured neurons and other cells p35 has been also found in the cell nucleus (Nikolic et al., 1996; Qu et al., 2002; Gong et al., 2003; Fu et al., 2006). Our BiFC data strongly support this nuclear location (Fig. $3 B$ ). In this context, it is significant that of the three known activators of Cdk5 only nuclear p35 demonstrates cell cycle suppression ability. The closely related p39 protein is capable of compensating for developmental p35 deficiency in vivo (Ko et al., 2001), but this redundancy does not appear to apply to the 
cell cycle activity. The failure to observe cell cycle suppression with p25 is also significant. Kim et al. (2008) recently have reported that p 25 induces neuronal cell cycle reentry through stimulating Cdk5 phosphorylation of HDAC1 and the induction of DNA damage. Many of the effects reported in this study occurred over a period of weeks, suggesting that chronic Cdk5 activity in the nucleus might have a very different outcome than shorter, acute episodes such as those we have studied here.

It is noteworthy that, in certain cell types, E2F1 itself appears to function as a cell cycle suppressor (Field et al., 1996). Indeed, our laboratory has explicitly argued that E2F1 acts as a cell cycle suppressor in neurons (Wang et al., 2007). This would appear to be at odds with the model presented in Figure 9. We have begun to resolve the apparent inconsistency by monitoring the levels of other E2F family members. We find E2F3 levels are dramatically upregulated in E2f1 ${ }^{-1-}$ brains compared with other E2Fs (supplemental Fig. 5, available at www.jneurosci.org as supplemental material). Figure 5, $D$ and $H$, shows that the nuclear E2F3 level in E2 $\mathrm{fl}^{-1-}$ were increased $\sim 15$ times compared with wild type. This suggests that elevated levels of E2F3 are the motive force behind the observed neuronal cell cycle events in the E2f1 ${ }^{-/-}$brain. Thus, E2F1 acts as a cell cycle suppressor by a feedback mechanism that normally keeps the levels of E2F3 low. The implication is that the failure of cell cycle suppression is indirect and mediated by E2F3.

It is telling that the complexes we describe differ dramatically depending on whether the players assemble in the nucleus or the cytoplasm. Our data show that Cdk5, p35, DP1, and E2F1 can bind each other as heterodimers in all combinations and in all locations. This means that each protein has a potential binding site for each of the other three proteins. Yet, when all four are present at the same time, Cdk5 and DP1 apparently compete to bind a p35-E2F1 complex. In the cytoplasm, DP1 keeps Cdk5 out of the complex, resulting in a DP1-p35-E2F1 trimer. In the nucleus, the situation reverses; Cdk5 keeps DP1 out of the complex to make a Cdk5-p35-E2F1 trimer (Fig. 9).

Our findings have implications for neurodegenerative diseases in general and Alzheimer's disease in particular. Cdk5 has been demonstrated to be an effective tau kinase. Since hyperphosphorylated tau is a major constituent of the neurofibrillary tangles found in Alzheimer's and other dementias, inhibition of Cdk5 activity has been proposed as a therapeutic approach to slowing the advance of the disease (Camins et al., 2006; Gong and Iqbal, 2008; Kaminosono et al., 2008). Kinase inhibition may prove effective in blocking the formation of tangles, but our results suggest caution in this approach. Cell cycle reentry is a common prelude to neurodegeneration in a variety of conditions. As nuclear Cdk5 acts as a cell cycle suppressor both in vivo and in vitro, it would follow that this characteristic of Cdk5 may be worth promoting, rather than inhibiting. Thus, any strategy aimed at blocking Cdk5 activity as a means of combating neurodegenerative disease should be carefully monitored for either direct or indirect effects on the levels or location of the Cdk5 protein. Treatments that lower overall protein levels or encourage nuclear emigration may produce unexpected and possibly unwanted consequences for the CNS.

\section{References}

Bandara LR, Buck VM, Zamanian M, Johnston LH, La Thangue NB (1993)

Functional synergy between DP-1 and E2F-1 in the cell cycle-regulating transcription factor DRTF1/E2F. EMBO J 12:4317-4324.

Camins A, Verdaguer E, Folch J, Canudas AM, Pallàs M (2006) The role of CDK5/P25 formation/inhibition in neurodegeneration. Drug News Perspect 19:453-460.
Cao L, Faha B, Dembski M, Tsai LH, Harlow E, Dyson N (1992) Independent binding of the retinoblastoma protein and p107 to the transcription factor E2F. Nature 355:176-179.

Cheung ZH, Ip NY (2007) The roles of cyclin-dependent kinase 5 in dendrite and synapse development. Biotechnol J 2:949-957.

Cicero S, Herrup K (2005) Cyclin-dependent kinase 5 is essential for neuronal cell cycle arrest and differentiation. J Neurosci 25:9658-9668.

Cruz JC, Tsai LH (2004) A Jekyll and Hyde kinase: roles for Cdk5 in brain development and disease. Curr Opin Neurobiol 14:390-394.

den Elzen N, Pines J (2001) Cyclin A is destroyed in prometaphase and can delay chromosome alignment and anaphase. J Cell Biol 153:121-136.

Field SJ, Tsai FY, Kuo F, Zubiaga AM, Kaelin WG Jr, Livingston DM, Orkin SH, Greenberg ME (1996) E2F-1 functions in mice to promote apoptosis and suppress proliferation. Cell 85:549-561.

Fu X, Choi YK, Qu D, Yu Y, Cheun NS, Qi RZ (2006) Identification of nuclear import mechanisms for the neuronal Cdk5 activator. J Biol Chem 282:39014-39021.

Gilmore EC, Ohshima T, Goffinet AM, Kulkarni AB, Herrup K (1998) Cyclin-dependent kinase 5-deficient mice demonstrate novel developmental arrest in cerebral cortex. J Neurosci 18:6370-6377.

Gong CX, Iqbal K (2008) Hyperphosphorylation of microtubule-associated protein tau: a promising therapeutic target for Alzheimer disease. Curr Med Chem 15:2321-2328.

Gong X, Tang X, Wiedmann M, Wang X, Peng J, Zheng D, Blair LA, Marshall J, Mao Z (2003) Cdk5-mediated inhibition of the protective effects of transcription factor MEF2 in neurotoxicity-induced apoptosis. Neuron 38:33-46

Guidato S, McLoughlin DM, Grierson AJ, Miller CC (1998) Cyclin D2 interacts with Cdk5 and modulates cellular Cdk5/p35 activity. J Neurochem 70:335-340.

Hawasli AH, Bibb JA (2007) Alternative roles for Cdk5 in learning and synaptic plasticity. Biotechnol J 2:941-948.

Herrup K, Yang Y (2007) Cell cycle regulation in the postmitotic neuron: oxymoron or new biology? Nat Rev Neurosci 8:368-378.

Hu CD, Kerppola TK (2003) Simultaneous visualization of interactions between multiple proteins in living cells using multicolor bimolecular fluorescence complementation analysis. Nat Biotechnol 21:539-545.

Hu CD, Chinenov Y, Kerppola TK (2002) Visualization of interactions among bZIP and Rel family proteins in living cells using bimolecular fluorescence complementation. Mol Cell 9:789-798.

Ishida H, Masuhiro Y, Fukushima A, Argueta JG, Yamaguchi N, Shiota S, Hanazawa S (2005) Identification and characterization of novel isoforms of human DP-1: DP- $1 \alpha$ regulates the transcriptional activity of E2F1 as well as cell cycle progression in a dominant-negative manner. J Biol Chem 280:24642-24648.

Ishida S, Huang E, Zuzan H, Spang R, Leone G, West M, Nevins JR (2001) Role for E2F in control of both DNA replication and mitotic functions as revealed from DNA microarray analysis. Mol Cell Biol 21:4684-4699.

Kaminosono S, Saito T, Oyama F, Ohshima T, Asada A, Nagai Y, Nukina N, Hisanaga S (2008) Suppression of mutant Huntingtin aggregate formation by Cdk5/p35 through the effect on microtubule stability. J Neurosci 28:8747-8755.

Kerppola TK (2006) Visualization of molecular interactions by fluorescence complementation. Nat Rev Mol Cell Biol 7:449-456.

Kim D, Frank CL, Dobbin MM, Tsunemoto RK, Tu W, Peng PL, Guan JS, Lee BH, Moy LY, Giusti P, Broodie N, Mazitschek R, Delalle I, Haggarty SJ, Neve RL, Lu Y, Tsai LH (2008) Deregulation of HDAC1 by p25/Cdk5 in neurotoxicity Neuron 10:803-817.

Ko J, Humbert S, Bronson RT, Takahashi AB, Kulkari AB, Li E, Tsai LH (2001) p35 and p39 are essential for cyclin-dependent kinase 5 function during neurodevelopment. J Neurosci 21:6758-6771.

Kotake Y, Nakayama K, Ishida N, Nakayama KI (2005) Role of serine 10 phosphorylation in p 27 stabilization revealed by analysis of p 27 knock-in mice harboring a serine 10 mutation. J Biol Chem 280:1095-1102.

Krek W, Ewen ME, Shirodkar S, Arany Z, Kaelin WG Jr, Livingston DM (1994) Negative regulation of the growth-promoting transcription factor E2F-1 by a stably bound cyclin A-dependent protein kinase. Cell 78:161-172.

Lee MH, Nikolic M, Baptista CA, Lai E, Tsai LH, Massagué J (1996) The brain-specific activator p35 allows Cdk 5 to escape inhibition by p27Kip in neurons. Proc Natl Acad Sci U S A 93:3259-3263.

Meyerson M, Enders GH, Wu CL, Su LK, Gorka C, Nelson C, Harlow E, Tsai 
LH (1992) A family of human cdc2-related protein kinases. EMBO J 11:2909-2917.

Mudryj M, Devoto SH, Hiebert SW, Hunter T, Pines J, Nevins JR (1991) Cell cycle regulation of the E2F transcription factor involves an interaction with cyclin A. Cell 65:1243-1253.

Müller H, Bracken AP, Vernell R, Moroni MC, Christians F, Grassilli E, Prosperini E, Vigo E, Oliner JD, Helin K (2001) E2Fs regulate the expression of genes involved in differentiation, development, proliferation, and apoptosis. Genes Dev 15:267-285.

Nikolic M, Dudek H, Kwon YT, Ramos YF, Tsai LH (1996) The cdk5/p35 kinase is essential for neurite outgrowth during neuronal differentiation. Genes Dev 10:816-825.

Nikolic M, Chou MM, Lu W, Mayer BJ, Tsai LH (1998) The p35/Cdk5 kinase is a neuron-specific Rac effector that inhibits Pak1 activity. Nature 395: $194-198$.

Patrick GN, Zukerberg L, Nikolic M, de la Monte S, Dikkes P, Tsai LH (1999) Conversion of p 35 to $\mathrm{p} 25$ deregulates Cdk5 activity and promotes neurodegeneration. Nature 402:615-622.

Qu D, Li Q, Lim HY, Cheung NS, Li R, Wang JH, Qi RZ (2002) The protein SET binds the neuronal Cdk5 activator p35 ${ }^{\text {nck5a }}$ and modulates Cdk5/ p35 ${ }^{\text {nck5a }}$ activity. J Biol Chem 277:7324-7332.

Ren B, Cam H, Takahashi Y, Volkert T, Terragni J, Young RA, Dynlacht BD (2002) E2F integrates cell cycle progression with DNA repair, replication, and $\mathrm{G}_{2} / \mathrm{M}$ checkpoints. Genes Dev 16:245-256.

Saito T, Nakatsuji N (2001) Efficient gene transfer into the embryonic mouse brain using in vivo electroporation. Dev Biol 240:237-246.

Spinella MJ, Freemantle SJ, Sekula D, Chang JH, Christie AJ, Dmitrovsky E (1999) Retinoic acid promotes ubiquitination and proteolysis of cyclin D1 during induced tumor cell differentiation. J Biol Chem 274:22013-22018.
Susakí E, Nakayama K, Nakayama KI (2007) Cyclin D2 translocates p27 out of the nucleus and promotes its degradation at the G0-G1 transition. Mol Cell Biol 27:4626-4640.

Tarricone C, Dhavan R, Peng J, Areces LB, Tsai LH, Musacchio A (2001) Structure and regulation of the CDK5-p25nck5a complex. Mol Cell 8:657-669.

Trimarchi JM, Lees JA (2002) Sibling rivalry in the E2F family. Nat Rev Mol Cell Biol 3:11-20.

Tsai LH, Delalle I, Caviness VS Jr, Chae T, Harlow E (1994) p35 is a neuralspecific regulatory subunit of cyclin-dependent kinase 5. Nature 371: $419-423$.

van den Heuvel S, Harlow E (1993) Distinct roles for cyclin-dependent kinases in cell cycle control. Science 262:2050-2054.

Wang L, Wang R, Herrup K (2007) E2F1 works as a cell cycle suppressor in mature neurons. J Neurosci 27:12555-12564.

Xiong Y, Zhang H, Beach D (1992) D type cyclins associate with multiple protein kinases and the DNA replication and repair factor PCNA. Cell 71:505-514.

Yang Y, Mufson EJ, Herrup K (2003) Neuronal cell death is preceded by cell cycle events at all stages of Alzheimer's disease. J Neurosci 23:2557-2563.

Yang Y, Varvel NH, Lamb BT, Herrup K (2006) Ectopic cell cycle events link human Alzheimer's disease and amyloid precursor protein transgenic mouse models. J Neurosci 26:775-784.

Zhang H, Xiong Y, Beach D (1993) Proliferating cell nuclear antigen and p21 are components of multiple cell cycle kinase complexes. Mol Biol Cell 4:897-906.

Zhang J, Cicero SA, Wang L, Romito-Digiacomo RR, Yang Y, Herrup K (2008) Nuclear localization of Cdk5 is a key determinant in the postmitotic state of neurons. Proc Natl Acad Sci U S A 105:8772-8777. 